\title{
Importance of capturing heliospheric variability for studies of thermospheric vertical winds
}

\author{
Erdal Yiğit, ${ }^{1,2}$ Aaron J. Ridley, ${ }^{1}$ and Mark B. Moldwin ${ }^{1}$ \\ Received 4 February 2012; revised 21 May 2012; accepted 22 May 2012; published 6 July 2012.
}

[1] Using the Global Ionosphere Thermosphere Model with observed real-time heliospheric input data, the magnitude and variability of thermospheric neutral vertical winds are investigated. In order to determine the role of variability in the Interplanetary Magnetic Field (IMF) and solar wind density on the neutral wind variability, the heliospheric input data are smoothed. The effects of smoothing the IMF and solar wind and density on the vertical winds are simulated for the cases of no smoothing, 5-minute, and 12-minute smoothing. Various vertical wind acceleration terms, such as the nonhydrostatic acceleration, are quantified. Polar stereographic projections of the variabilities of vertical wind and ion flows are compared to highlight existing correlations. Overall, the smoother, that is, the less variable the IMF and solar wind parameters are, the weaker are the magnitude and the variability of the thermospheric vertical winds. Weaker IMF variability leads to smaller variability in ion flows, which in turn negatively impacts the variability and the magnitude of Joule heating. Small-scale temporal variation of the vertical wind acceleration, and thus the variability of the vertical wind, is dominated by the nonhydrostatic term that is controlled primarily by the temporal variation of the Joule heating, which in turn is related to ion flow variations that are shaped by the IMF in the high-latitude thermosphere. Wavelet analysis of the vertical wind data shows that gravity waves of $\sim 5$ and $\sim 10$-minute periods are more prominent when the model is run with high-resolution real-time IMF and solar wind data. Better capturing of the temporal variation of the IMF and solar wind parameters is crucial for modeling the variability and magnitude of thermospheric vertical winds.

Citation: Yiğit, E., A. J. Ridley, and M. B. Moldwin (2012), Importance of capturing heliospheric variability for studies of thermospheric vertical winds, J. Geophys. Res., 117, A07306, doi:10.1029/2012JA017596.

\section{Introduction}

[2] The thermosphere-ionosphere, which forms the portion of the upper atmosphere above the mesopause, is a highly energetic and variable region that is coupled to the magnetosphere above and to the lower and the middle atmosphere below. Besides the diurnal variations of the solar UV and EUV energy input that generate large-scale and lowfrequency local variations, there is substantial amount of small-scale high-frequency variation in the system that cannot be explained by considering solely the solar energy input. Variability of the upper atmosphere can be considered at various temporal and spatial scales. Not all scales can be captured by atmospheric models due to uncertainties in

\footnotetext{
${ }^{1}$ Department of Atmosphere, Oceanic and Space Sciences, University of Michigan, Ann Arbor, Michigan, USA.

${ }^{2}$ Space Sciences Laboratory, University of California, Berkeley, California, USA.

Corresponding author: E. Yiğit, Department of Atmosphere, Oceanic and Space Sciences, University of Michigan, 1429 Space Research Bldg., 2455 Hayward St., Ann Arbor, MI 48109-2143, USA. (erdal@umich.edu)

(C)2012. American Geophysical Union. All Rights Reserved. 0148-0227/12/2012JA017596
}

dynamical and thermal drivers and the characteristics of boundary conditions, and limitations in model temporal and spatial resolutions. Empirical models are typically used to represent the overall structure and variations of the atmosphere. However, observations indicate that there is a remarkable variability, for example, in ionospheric ion flows [Johnson and Heelis, 2005; Bristow, 2008], electric fields [Heppner et al., 1993; Matsuo et al., 2003; Kozelov et al., 2008], composition [Kil et al., 2011], Joule heating [Rodger et al., 2001], and vertical winds [Innis and Conde, 2001; Anderson et al., 2011] in the upper atmosphere that is missing in empirical models. The term variability is often used to describe the variations of the system that are not represented by empirical models. Understanding the characteristics and the sources of upper atmospheric variability is crucial for better interpreting observations and improving global models and empirical models that are implemented in them. Global models are great diagnostic tools, which can be used to investigate the interplay between the different sources and manifestations of variability. This paper focuses on the magnitude and temporal variability of thermospheric vertical winds. 
[3] Thermospheric vertical winds play an important role for the momentum and energy budget and compositional structure [Johnson et al., 1995] of the upper atmosphere. A number of observations demonstrate that the thermosphereionosphere is permeated continuously by large vertical winds [Spencer et al., 1976; Conde and Dyson, 1995; Rees et al., 1984a; Wardill and Jacka, 1986; Price et al., 1995; Innis et al., 1996, 1999; Ishii et al., 2001; Anderson et al., 2011] and thus these large vertical winds cannot be considered as minor disturbances in the upper atmosphere. Their magnitude and variability are still underestimated in general circulation models (GCMs) [Conde and Dyson, 1995] and it is thus crucial for the geophysics community to investigate possible reasons of underestimating vertical wind magnitude and variability and what mechanisms control their magnitude and variability in space-time.

[4] Recently, Anderson et al. [2011] have conducted Doppler measurements of thermospheric vertical winds using Fabry-Perot spectrometers in the Southern Hemisphere high-latitudes. They observed that downward vertical winds of more than $100 \mathrm{~m} \mathrm{~s}^{-1}$ can occasionally occur. Also, they found that there was a clear correlation between auroral activity and vertical winds and large vertical winds were observed during times of strong horizontal wind divergence, suggesting that the Burnside relation [Burnside et al., 1981] applied to a large extent. Their observations were performed at nighttime in two consecutive days, 7-8 April 2008, when the observed Interplanetary Magnetic Field (IMF) had relatively small magnitudes but significant small-scale temporal variability. Their measurements demonstrated highly variable vertical winds. The goal of this current paper is to investigate the modeling and theoretical aspects of the magnitude, variability, and dynamical mechanisms for vertical winds using realistic observed real-time geomagnetic and solar activity during the period Anderson et al. [2011] made their observations. The Global Ionosphere Thermosphere Model (GITM) will be used for this purpose to conduct diagnostic studies of vertical acceleration, high-latitude Joule and auroral heating, and high-frequency wave structures.

[5] Section 2 describes briefly the Global Ionosphere Thermosphere Model; section 3 summarizes the model setup and configuration of runs with the details of model input for the IMF and solar wind parameters. Results are presented in section 4 for the temporal variations of the neutral vertical winds, ion and neutral flows, and the variability of vertical winds in the auroral thermosphere. Finally wave structures will be investigated. Discussion and conclusions are given in section 5 .

\section{Global Ionosphere Thermosphere Model}

\subsection{General Characteristics}

[6] The Global Ionosphere Thermosphere Model (GITM) [Ridley et al., 2006] is a three-dimensional nonlinear nonhydrostatic first-principle general circulation model (GCM) extending from $\sim 100$ to about $650 \mathrm{~km}$, depending on the heliospheric activity. It is based on an altitude grid with approximately one third scale height separation between the consecutive altitude levels, consisting of 50 levels. The model grid resolution is flexible and the time step is typically $2-4 \mathrm{~s}$.

\subsection{Nonhydrostatic Effects}

[7] GITM solves the vertical momentum equation explicitly, enabling the model to account for nonhydrostatic acceleration of the vertical wind. A number of traditional GCMs modeling the thermosphere are based on the assumption of hydrostatic equilibrium [Fuller-Rowell and Rees, 1980; Dickinson et al., 1981; Roble et al., 1988; Richmond et al., 1992; Wang et al., 1999; Yigit et al., 2009]. Provided that the hydrostatic balance is valid, the upward directed pressure force exactly balances the downward directed gravitational acceleration:

$$
\frac{\partial p}{\partial r}=-\rho g,
$$

where $p$ is pressure, $\rho$ is neutral mass density, $r=R_{E}+z$ is the radial distance from the center of the Earth with $R_{E}$ being the Earth's radius and $z$ the altitude above the surface, and $g$ is the gravitational acceleration that varies as a function of altitude. Vertical winds are then derived by determining the time rate of change of a constant pressure level. This is often adequate outside the auroral oval, where rapid heating is relatively uncommon. In the auroral thermosphere, however, the atmosphere can depart significantly from a hydrostatic balance primarily because of intensive energy and momentum flux deposition of magnetospheric origin and also, to some extent, disturbances of lower atmospheric origin. Thus, in GITM, the hydrostatic balance (1) is replaced by the explicit form of the vertical momentum equation [Yiğit and Ridley, 2011a, equation (2)]:

$$
\frac{D w_{n}}{D t}=-\frac{1}{\rho} \frac{\partial p}{\partial r}+g+a_{c o r}+a_{i n}+a_{n n}+a_{s p h},
$$

where $w_{n}$ is the neutral vertical wind, $D / D t=\partial / \partial t+\mathbf{u} \cdot \nabla$ is the advective derivative with neutral wind velocity $\mathbf{u}=$ $\left(u_{n}, v_{n}, w_{n}\right), a_{c o r}$ includes acceleration due to Coriolis and centrifugal effects, $a_{i n}$ is vertical ion drag, $a_{n n}$ is neutral friction, and $a_{s p h}$ is the sphericity term. The nature of these terms are discussed in some detail in the work by Ridley et al. [2006] and Yiğit and Ridley [2011a].

\subsection{Model Resolution}

[8] Model resolution is variable. The horizontal domain is discretized in terms of blocks, where each block consists of $n \times m$ longitude-latitudes cells. Yigit and Ridley [2011b] have systematically tested the impact of horizontal grid resolution on the high-latitude Joule heating, increasing the longitude-latitude resolution from $5^{\circ} \times 5^{\circ}$ to $2.5^{\circ} \times 0.3125^{\circ}$. In the present study, we employ a fixed uniform resolution of $2.5^{\circ} \times 2.5^{\circ}$ for the simulations that investigate the importance of IMF variations for the vertical wind variability.

\subsection{Boundary Conditions}

[9] GITM is initialized using the International Reference Ionosphere and the Mass Spectrometer - Incoherent Scatter model (MSIS) [Hedin, 1991]. MSIS is used to determine the lower boundary values for the major advective species $\left(\mathrm{N}_{2}, \mathrm{O}_{2}, \mathrm{O}\right.$, and $\left.\mathrm{N}\right)$. NO is allowed to have a constant gradient 
boundary condition (NO is not included in MSIS). The temperature is also set by the MSIS model. All winds are set to zero at the bottom boundary, including ion flows. The ion densities are allowed to have a constant gradient, while the electron and ion temperatures are set to the neutral temperatures.

[10] The upper boundary conditions are much more complicated. Simplistically, the neutral densities are allowed to have a constant gradient (essentially hydrostatic). The winds and temperatures have a continuous value (i.e., zero gradient). The ion densities can have a decreasing density but not too large of an increase in density (since this can be a large source of plasma, which causes too much inflow).

[11] The high-latitude magnetospheric potential and aurora are specified by the Weimer [1996] and Fuller-Rowell and Evans [1987] empirical models, respectively. The inputs to the Weimer potential model are the IMF $B_{y}$, IMF $B_{z}$, solar wind velocity and tilt angle of the dipole with respect to the ecliptic (specified by the date and time). The IMF is recorded with a $15-20 \mathrm{~s}$ time resolution. It is also propagated to the magnetosphere using a simple propagation scheme given the distance from the magnetosphere to the Advanced Composition Explorer (ACE) satellite, which measures the IMF and solar wind, and the solar wind velocity [e.g., Ridley et al., 2001]. Better methods of propagation exist [e.g., Weimer et al., 2002], but a constant $+/-10$ minute time offset in the model results will not effect the results much at all, and do not really matter unless we are comparing directly to data, especially onset of features. In this study, discontinuities or sudden onsets of features are not explored; it is investigated how variability, in general, affects the thermospheric state. The Fuller-Rowell and Evans [1987] model is driven by the hemispheric power index, which is recorded by National Oceanic and Atmospheric Administration satellites. The hemispheric power is reported in every approximately $45-50$ minutes for each satellite (time to orbit from one pole to the other), which can result in a highly non-uniform temporal cadence for the hemispheric power specification. GITM linearly interpolates between the different measurements of the hemispheric power to allow a "continuous" specification.

[12] Within the code, an input parameter is specified to control how often the electrodynamics are updated. In this study, $60 \mathrm{~s}$ is utilized. Since GITM does not take uniform time steps, when GITM crosses a $60 \mathrm{~s}$ boundary (i.e., $60 \mathrm{~s}$, $120 \mathrm{~s}, 180 \mathrm{~s}$, etc), a new potential pattern and auroral pattern are generated from the empirical models, as described above. This exact same technique is used whether the IMF. It is represented in the model by either samples at 15-20 s or by average values, e.g., 12-minute averages. The empirical models used within this study do not contain any real variability - they are heavily averaged models. Allowing the potential to change dramatically, forces the ionosphere to have variability, which may or may not be similar to that observed by Bristow [2008]. This will be discussed further within the paper, but briefly, the ion flows will change most dramatically in regions in which there are strong gradients in the flow. For example, near the convection reversal boundary $(\mathrm{CRB})$, a small change in the IMF can reverse the sign of the ion flow near the $\mathrm{CRB}$, but might not change the ion flows in the polar cap by more than a couple of percent.

\section{Model Setup and Configuration of Runs}

\subsection{Interplanetary Magnetic Field and Solar Wind Parameters}

[13] Figure 1 shows the observed real-time variations of the Interplanetary Magnetic Field (IMF) components $B_{x}, B_{y}, B_{z}$, and solar wind density and speed from 6 to 9 April 2008 observed by the National Aeronautics and Space Administration (NASA) Advanced Composition Explorer (ACE) Satellite. All data have typically 15-20 s time resolution. All parameters demonstrate strong temporal fluctuations, but overall both IMF and the solar wind are relatively quiescent with $\left|B_{z}\right| \leq 5 \mathrm{nT}$. The year of 2008 was overall very quiet in terms of geomagnetic and solar activities.

[14] Figure 2 shows the IMF $B_{z}$ between 7 April 1600 UT and 8 April 0000 UT in more detail. This interval was focused on in the work by Anderson et al. [2011]. Note that their Figure 3 shows the IMF $B_{z}$ in 4-minute and 1-hour averages, while in the modeling studies to be performed in the present paper, much high-time resolution IMF and solar wind data are used. Close investigation shows that the observed IMF $B_{z}$ (black solid line) has rapid variations of less than a minute. In order to control the variability of the heliosphere, the IMF and solar wind input data shown in Figure 1 are smoothed by calculating the 2-, 5-, and 12-minute running means and the variations are overplotted by blue, yellow, and red solid lines. The first two hours of this period will be studied in more detail in this paper and no smoothing, 5-minute, and 12-minute smoothing cases will be focused on. As stronger smoothing is applied to the input data, the small-scale temporal variability of $B_{z}$ decreases. The magnitude of the variability of a given parameter depends highly on the time interval of choice, in other words, on how the data are binned. This is demonstrated in Figure 3, where the variability of the IMF $B_{z}$ is evaluated in 15-minute (solid) and 1-hour (dotted) bins for the three model simulations of no smoothing, 5-minute and 12-minute smoothing. The IMF variability drops dramatically with increasing degree of smoothing both in 15-minute and 1-hour binning cases. For example, at 2100 UT in the 15-minute binning, the peak variability is around $2.6 \mathrm{nT}$ in the no smoothing simulation, while it decreases to $\sim 2 \mathrm{nT}$ in 5-minute smoothing and then to about $1.5 \mathrm{nT}$ in the 12-minute smoothing case.

[15] These figures demonstrate that although the solar and geomagnetic activity are overall low, there is a lot of smallscale temporal variability in the geospace system, which can be studied using the capabilities of GITM. These rapid fluctuations are expected to impact the thermosphere-ionosphere, in particular, the vertical wind variability. In the following sections, the importance of capturing small-scale heliospheric variability for the magnitude and variability of vertical winds is studied.

\subsection{Model Simulations}

[16] GITM has been run from 6 to 8 April 2008 with the realistic variations of auroral activity, the IMF and 

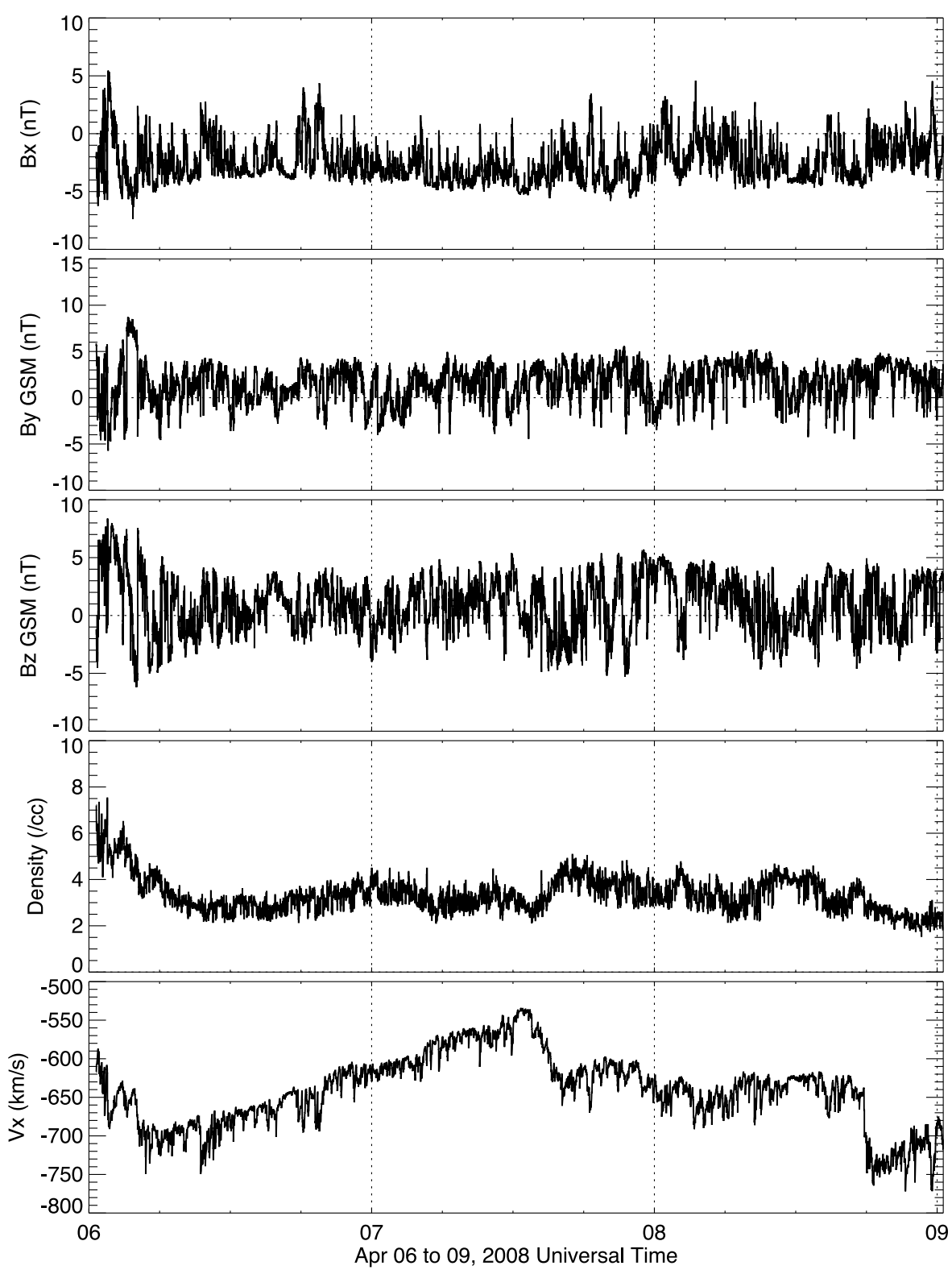

Figure 1. Global Ionosphere Thermosphere Model (GITM) input: Heliospheric variations for interplanetary magnetic field (IMF) components, and solar wind density and speed from 6 to 9 April 2008.

solar wind parameters for the cases of (1) no smoothing, (2) 5-minute smoothing, and (3) 12-minute smoothing of the IMF and solar wind parameters presented in Figure 2 from 1600 to $2400 \mathrm{UT}$ on 7 April. In simulations where the input data have been smoothed, i.e., (2) and (3), only the IMF and solar wind parameters are smoothed to isolate the different effects from each other. In other words, the same auroral activity data (not shown) have been used for all three simulations. Model data are studied between 1600 and 1800 UT, based on 1-minute data outputs.

\section{Results}

\subsection{Universal Time Variation of Vertical Wind}

[17] Figure 4 presents the nighttime universal time (UT) variations of vertical acceleration, vertical wind, auroral (QA) and Joule heating $\left(Q_{J}\right)$, and the horizontal wind divergence for a representative high-latitude location $\left(68.75^{\circ} \mathrm{S}, 78.75^{\circ} \mathrm{E}\right)$ at $285 \mathrm{~km}$ between 1600 and $1800 \mathrm{UT}$ on 7 April 2008. This altitude is close to the level where Fabry Perot measurements are made [Anderson et al., 2011]. In the first panel, red, blue, and black colors denote the vertical ion drag $\left(a_{i o n}\right)$, Coriolis force $\left(a_{c o r}\right)$, and nonhydrostatic acceleration $\left(a_{n h y d}\right)$ in $\mathrm{m} \mathrm{s}^{-1}$, while solid, dotted, and dashed lines are for the no smoothing, 5-minute and 12minute smoothing cases, respectively. We define nonhydrostatic acceleration as any departure from a perfect hydrostatic equilibrium in which the upward directed pressure force does not exactly balance the downward directed gravitational acceleration, i.e.,

$$
\frac{\partial p}{\partial r} \neq-\rho g
$$




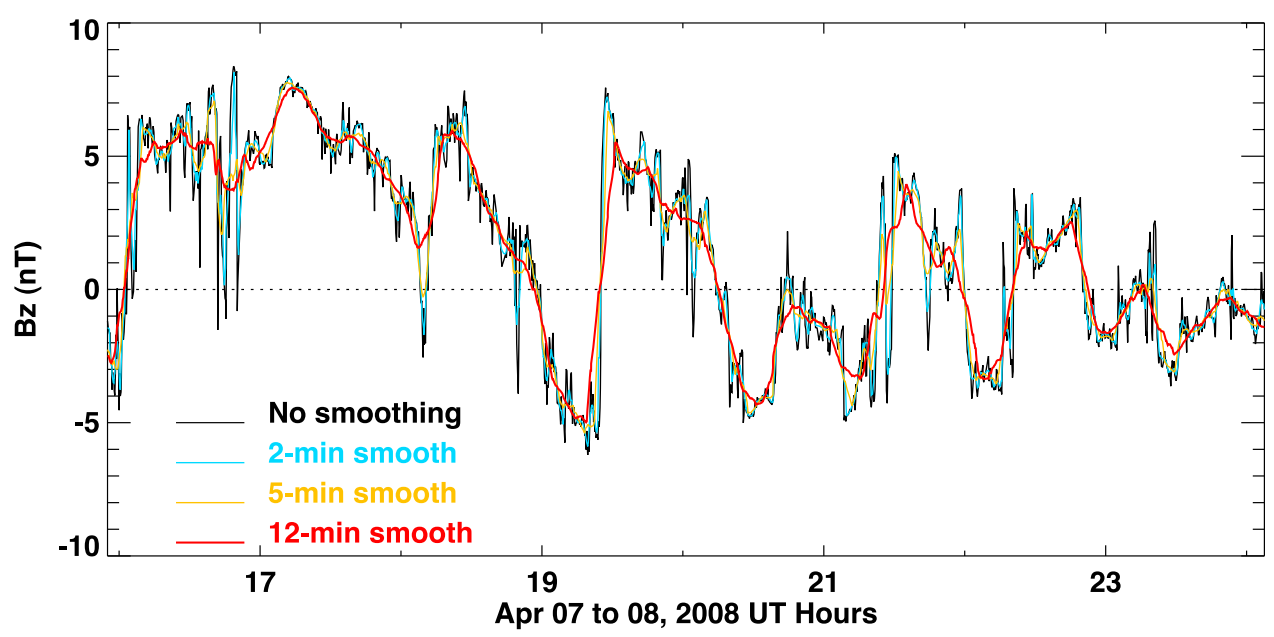

Figure 2. IMF $B_{z}$ input in four different model simulations in which IMF, solar wind and density have been smoothed differently. Solid line represents the no smoothing case, while blue, yellow, and red lines show the data with 2-, 5-, and 12-minute running means in the time interval of 1600 UT to 2400 UT on 7 April 2008.

[18] Thus, superposing the upward directed pressure gradient on the downward directed gravitational acceleration, one gets the magnitude and direction of the nonhydrostatic acceleration:

$$
a_{\text {nhyd }}=\frac{\partial p}{\partial r}+\rho g .
$$

[19] By assuming idealized IMF variations within GITM, Yiğit and Ridley [2011a] demonstrated that the imbalance between the pressure force and the gravitational force plays a significant role for the magnitude and variability of thermospheric vertical winds. In this study, we use observed real-time IMF variations as described in section 3.1. Vertical ion drag and Coriolis accelerations vary relatively little in the two-hour period shown here and they are not affected by smoothing of the IMF and the solar wind parameters, either.
On the other hand, variations of $a_{\text {nhyd }}$ with universal time are overall evident and highly depend on the smoothing of the IMF. Nonhydrostatic acceleration is weaker when the IMF is smoothed.

[20] The second panel in Figure 4 shows the neutral vertical winds in $\mathrm{m} \mathrm{s}^{-1}$. Large-scale as well as small-scale variations are seen as a function of universal time. Variability of the IMF clearly impacts the variability and the magnitude of the vertical winds. The no smoothing case, that is, the highest IMF variability case, produces overall the largest vertical wind variability and magnitude, while weaker IMF variability produced by stronger smoothing leads to less variability of vertical winds. Overall, $w_{n}$ varies between 5 and $-10 \mathrm{~m} \mathrm{~s}^{-1}$ in the presented two-hour period. In order to study vertical winds and their generation in the light of localized heat sources, Joule (blue) and auroral (black) heating in $\mathrm{K} \mathrm{s}^{-1}$ are presented in the third panel for the same time period. In the nighttime, Joule and auroral

\section{Apr 1600-2400 UT}

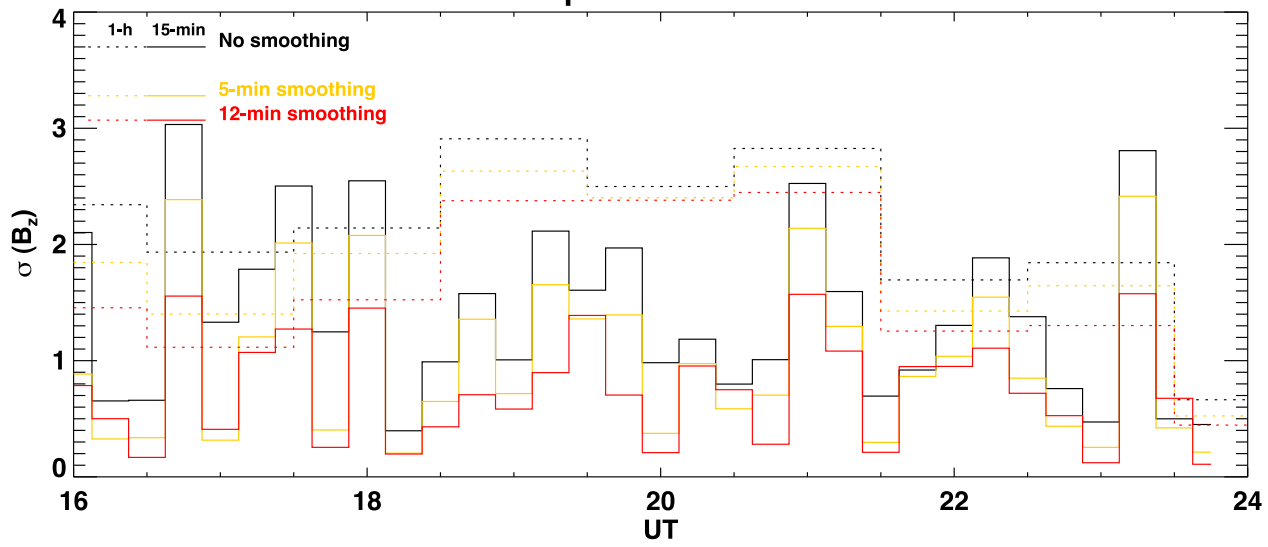

Figure 3. Temporal variability of the IMF $B_{z}$ from 1600 to 2400 UT on 7 April 2008 evaluated as standard deviations $\left(\sigma\left(B_{z}\right)\right)$ within 15- and 60-minute intervals. Black, yellow and red colors denote the no smoothing, 5-minute, and 12-minutes smoothing cases, respectively. Dotted and solid lines represent $\sigma\left(B_{z}\right)$ for the 15 - and 60 -minute time windows. 
$68.75^{\circ} \mathrm{S}, 78.75^{\circ} \mathrm{E}, 285 \mathrm{~km}, 7$ Apr $1600-1800 \mathrm{UT}$
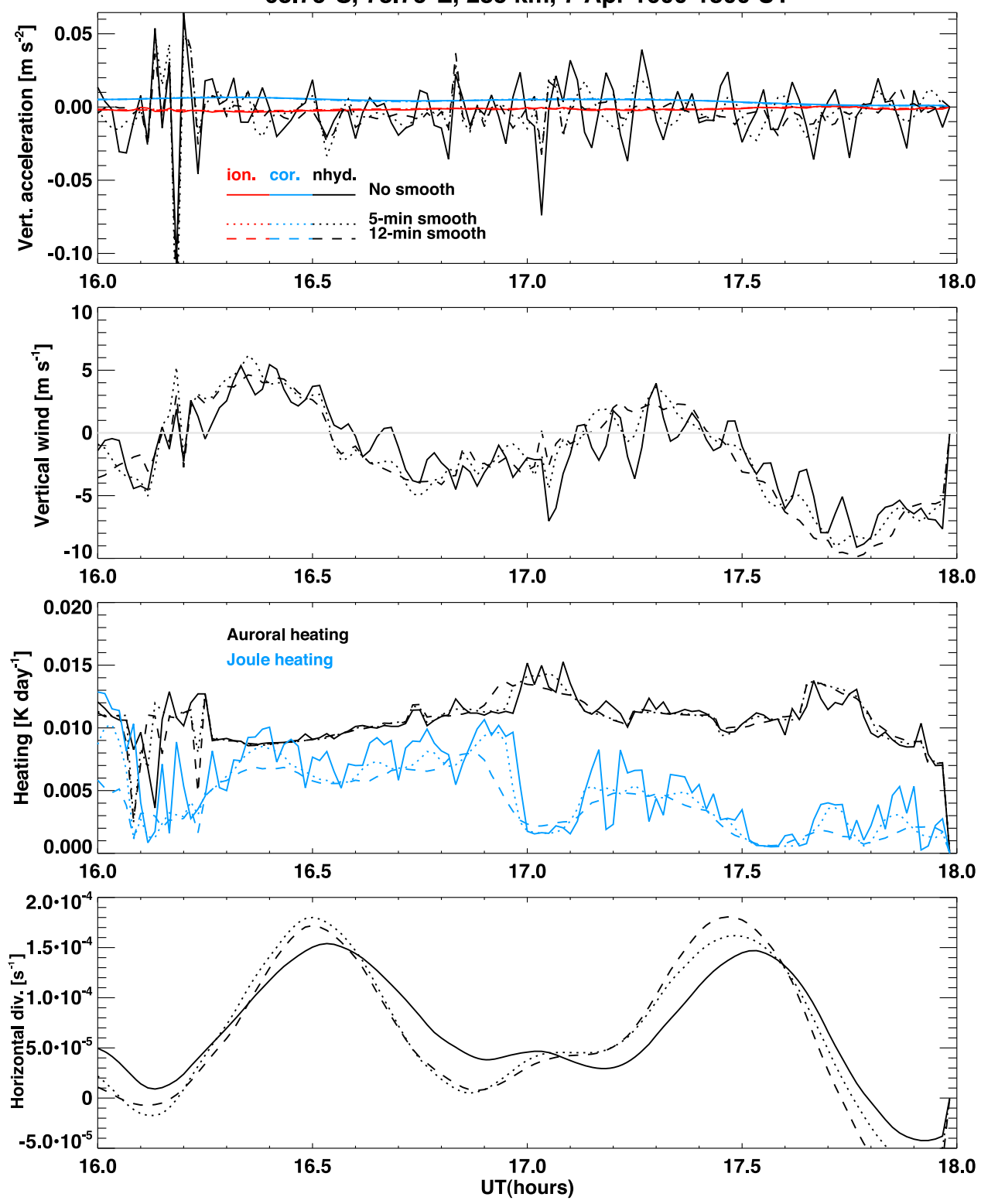

Figure 4. Two-hour variations at $68.75^{\circ} \mathrm{S}, 78.75^{\circ} \mathrm{E}$ and $285 \mathrm{~km}$ on 7 April 2008 of vertical acceleration, vertical wind, Joule and auroral heating, and horizontal divergence for the three model simulations with no smoothing, 5-minute, and 12-minute smoothing for the IMF, solar wind and density input data shown by solid dotted and dashed lines, respectively. In the first panel, red, blue, and black colors denote vertical wind acceleration due to vertical ion drag, Coriolis and centrifugal effects, and nonhydrostatic acceleration, respectively. In the third panel, black and blue lines represent auroral and Joule heating, respectively.

heating are the most dominant neutral gas heating processes because of lack of direct solar illumination. As the variability of the hemispheric power has been kept the same for the different runs of IMF and solar wind data smoothing, there are overall little changes in the auroral heating between the three simulations. Any resulting variation has to do with changes in the thermospheric structure, since this effects the energy deposition and how that energy actually heats the thermosphere (i.e., specific heat at constant volume and neutral density $\rho$ ). However, Joule heating is much more dependent on the IMF and solar wind conditions. This is because the IMF impacts the $\mathbf{E} \times \mathbf{B}$ ion drifts whose variations can modulate $Q_{J}$ dramatically. Variations of ion flows will be studied in more detail in section 4.2. For the no smoothing case, in which the IMF and high-resolution solar wind data are used, Joule heating has the largest temporal variability and magnitude. It has a very complex temporal variation with peak values of $\sim 0.01 \mathrm{~K} \mathrm{~s}^{-1}$ around 1600 and 1700 UT. Overall, Joule heating is weaker than auroral heating during the nighttime as it is more dependent on the electron density, which is largely controlled by solar illumination. It is important to note that Joule heating and auroral particle precipitation are coupled processes. Often, $E$ region electron density in the polar nighttime sector is 

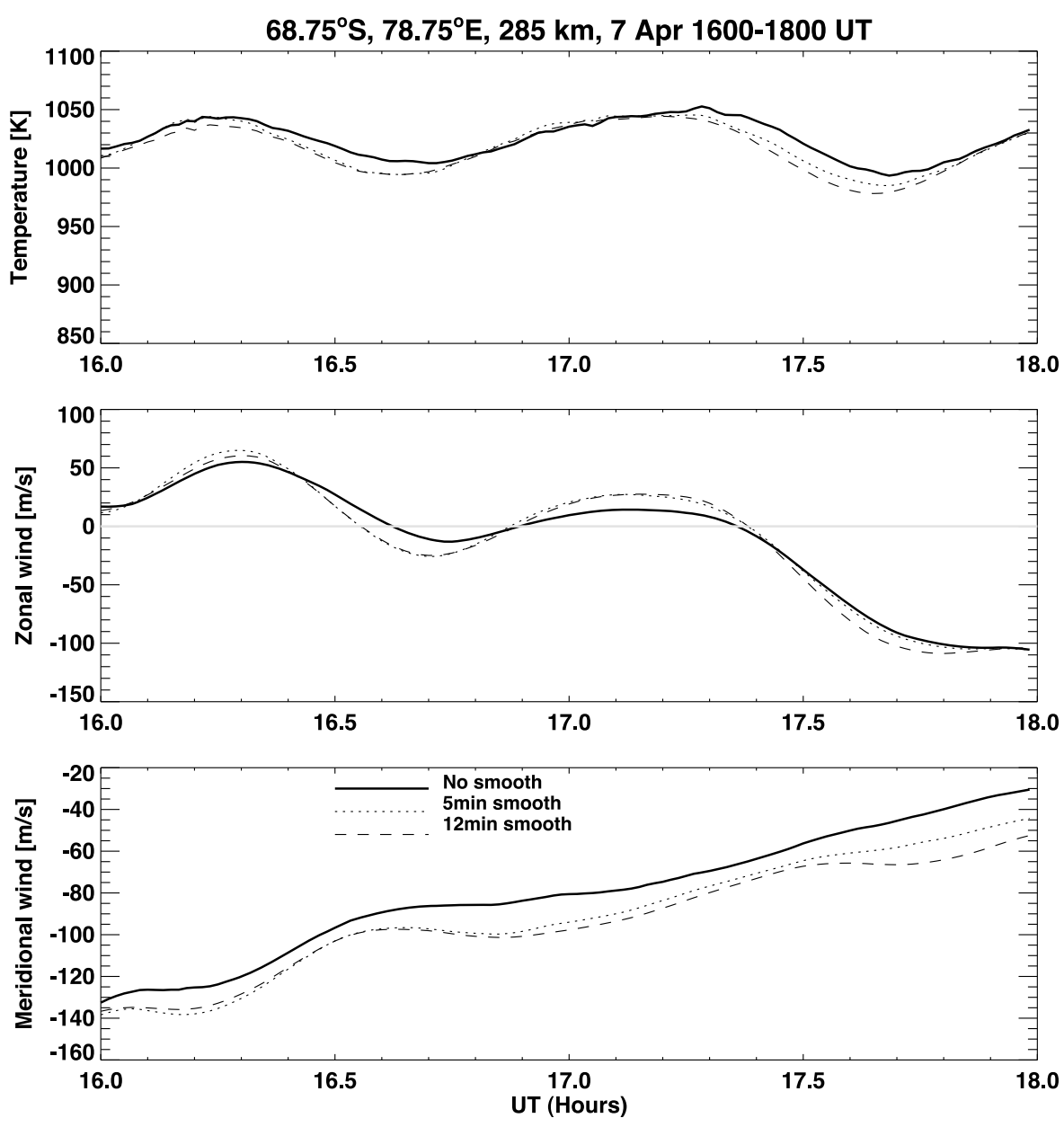

Figure 5. Two-hour variations at $68.75^{\circ} \mathrm{S}, 78.75^{\circ} \mathrm{E}$ and $285 \mathrm{~km}$ on 7 April 2008 of neutral temperature, zonal and meridional winds for the no smoothing, 5-minute and 12-minute smoothing cases denoted by solid, dotted and dashed lines, respectively.

enhanced by particle precipitation, and Joule heating can exceed particle heating.

[21] Divergence of horizontal neutral winds is an important dynamical process that can drive upwelling/downwelling via mass continuity. The fourth panel in Figure 4 shows the divergence of the horizontal neutral winds, $\nabla \cdot \mathbf{u}_{\mathbf{H}}$, in $\mathrm{s}^{-1}$. Seen on timescales of several minutes, $\nabla \cdot \mathbf{u}_{\mathbf{H}}$ varies more slowly than the modeled nonhydrostatic acceleration, the vertical wind, Joule and auroral heating terms. If the variation of the model data is analyzed on a larger timescale of an hour (i.e., large-scale temporal variation), it is noteworthy that the extrema of the horizontal wind divergence appear to coincide with the large-scale variations of the vertical wind. Comparison of the first three panels suggests that nonhydrostatic processes, vertical winds, Joule and auroral heating demonstrate similar small-scale temporal variations, which will be considered further in the rest of the paper.

\subsection{Variations of the Neutral and Ion Parameters}

[22] To further reconcile the variations of the neutral winds with the variations of the nonhydrostatic acceleration and the Joule and auroral heating presented in Figure 4, we next investigate the details of neutral wind and ion flow variations for the same period of time. Figure 5 shows the UT variations of neutral temperature, zonal and meridional winds at $285 \mathrm{~km}$ at $68.75^{\circ} \mathrm{S}$ and $78.75^{\circ} \mathrm{E}$ for the no smoothing, 5-minute and 12-minute smoothing simulations shown in black solid, dotted, and dashed lines. Additionally, the zero $\mathrm{m} \mathrm{s}^{-1}$ line is marked in grey. Overall, there is not a significant variation of the neutral parameters between the different model simulations. However, note that the no smoothing simulation produces overall slightly larger temperature values at this specific location. For example, around 17.5 UT, it is $20 \mathrm{~K}$ higher than the temperature in the 12-minute smoothing simulation.

[23] Figure 6 shows the zonal, meridional, and vertical ion speeds for the same two-hour period in the same manner as for the neutrals for the no smoothing, 5-minute, and 12-minute smoothing cases. There is a remarkable smallscale temporal variability in the ion flows with the largest magnitude and variability simulated by the no smoothing simulation. Ions are much faster than the neutrals, primarily owing to larger acceleration associated with the convection electric fields at the high-latitudes. The more the IMF and solar wind parameters are smoothed, the weaker and less variable are the ion flows. It is striking that the overall smallscale structure of the ion speed temporal variations resemble 

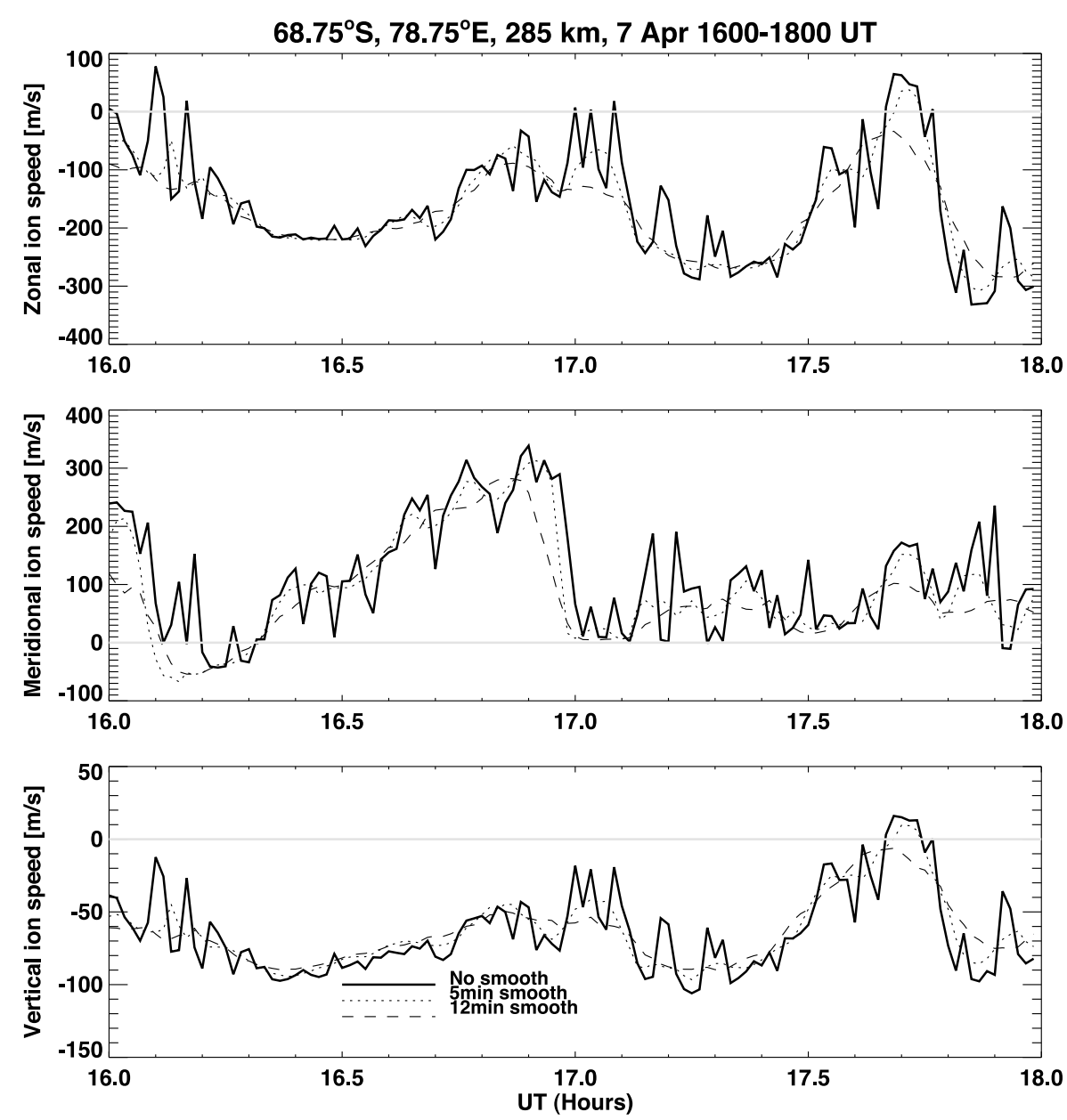

Figure 6. Two-hour variations at $68.75^{\circ} \mathrm{S}, 78.75^{\circ} \mathrm{E}$ and $285 \mathrm{~km}$ on 7 April 2008 of zonal, meridional, and vertical speeds for the no smoothing, 5-minute and 12-minute smoothing cases denoted by solid, dotted and dashed lines, respectively.

the temporal variations in the vertical winds, Joule heating and auroral heating, and the nonhydrostatic acceleration presented in Figure 4. These results support strong coupling between IMF variations, ionospheric ion flows and Joule heating.

\subsection{Vertical Wind Variability at High-Latitudes}

[24] Three-dimensional GCMs provide the advantage of investigating global pictures of atmospheric parameters. Figure 7 presents the Northern (Figures $7 \mathrm{a}$ and $7 \mathrm{~b}$ ) and Southern (Figures 7c and 7d) Hemisphere polar stereographic projections of neutral vertical wind variability in $\mathrm{m} \mathrm{s}^{-1}$ in color shading poleward of $45^{\circ} \mathrm{N} / \mathrm{S}$ at around $285 \mathrm{~km}$ for the two-hour period, 1600-1800 UT. The magnitude of temporal variability is evaluated in terms of the standard deviations, $\sigma$, over time using 1-minute model outputs in a two-hour window. The two-hour mean Joule heating $\left(Q_{J}\right)$ in $\mathrm{K} \mathrm{s}^{-1}$ is overplotted in red contour lines. The no smoothing and 12-minute cases are displayed in the left and right columns, respectively, for comparison. The high-latitude distributions are consistent with the results presented in the previous figures for a fixed Southern Hemisphere auroral latitude. In both hemispheres, the largest vertical wind variability is generated when the IMF has the largest variability, i.e., no smoothing case. Peak vertical wind variability, $\sigma_{w}$, is found at auroral latitudes at $0^{\circ}$ and $180^{\circ}$ longitude, where there is a significant amount of Joule heating. In both hemispheres, peaks of $\sigma_{w}$ coincide well with the regions of peak mean Joule heating. For example, in the Northern Hemisphere around $0^{\circ}$, in the no smoothing case $\sigma_{w} \sim 10 \mathrm{~m} \mathrm{~s}^{-1}$ and $Q_{J} \sim 0.09 \mathrm{~K} \mathrm{~s}^{-1}$ while in the 12-minute smoothing case these values are considerably smaller: $\sigma_{w} \sim 5-6 \mathrm{~m} \mathrm{~s}^{-1}$ and $Q_{J} \sim 0.07 \mathrm{~K} \mathrm{~s}^{-1}$. Similar systematic tendencies are seen in the Southern Hemisphere as well, however the patterns are displaced in longitude and latitude.

[25] Overall, the Northern and Southern Hemispheres are very asymmetric in the distribution and magnitude of vertical wind variability and two-hour mean Joule heating. The peak $\sigma_{w}$ in the Southern Hemisphere is up to $6 \mathrm{~m} \mathrm{~s}^{-1}$ and the peak mean $Q_{J}$ is about $0.06 \mathrm{~K} \mathrm{~s}^{-1}$ and are much less than in the Northern Hemisphere and occur in different places. The differences and the asymmetry between the Northern and Southern Hemispheres arise primarily because the geographic and the geomagnetic axes are offset and the amount of offset between the geographic and the geomagnetic poles are different in the different hemispheres. However, in both hemispheres the morphology of the distribution of $\sigma_{w}$ resembles closely the structure of the auroral 


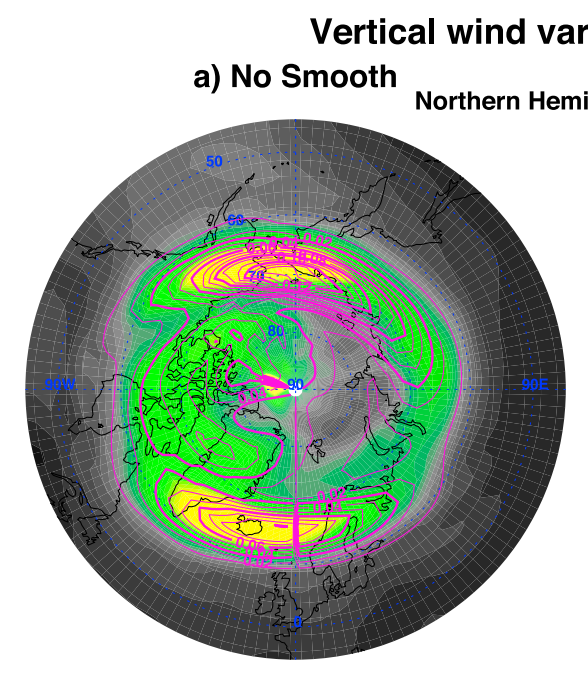

c) No Smooth

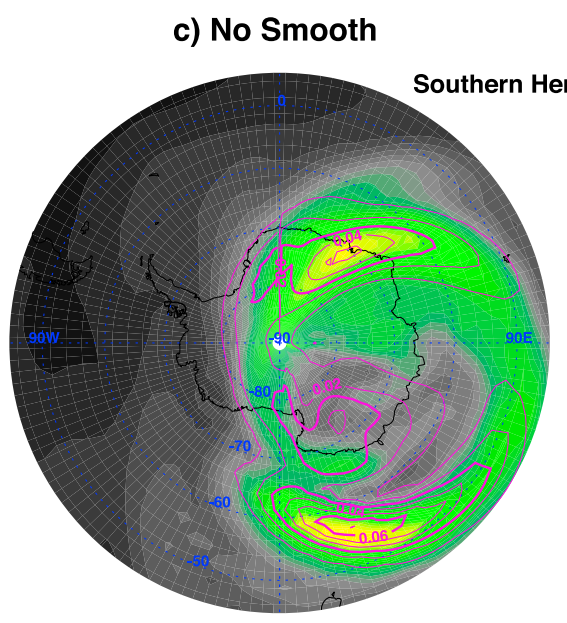

iability, 285 km

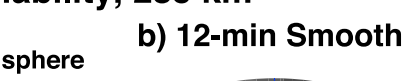

\author{
poter
}

Smooth

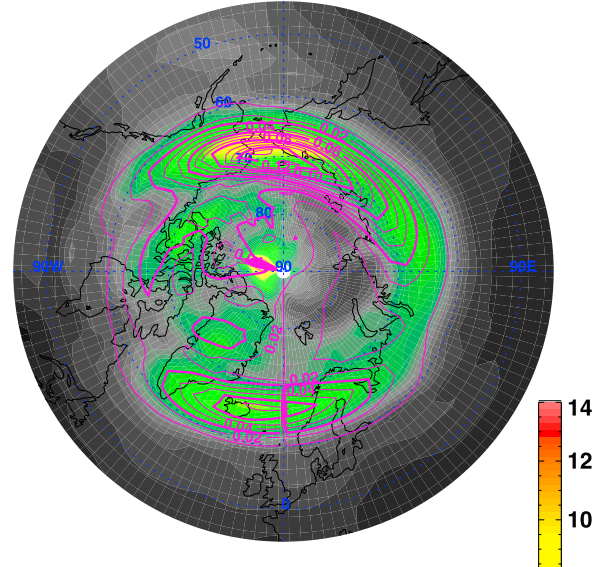

d) 12-min Smooth

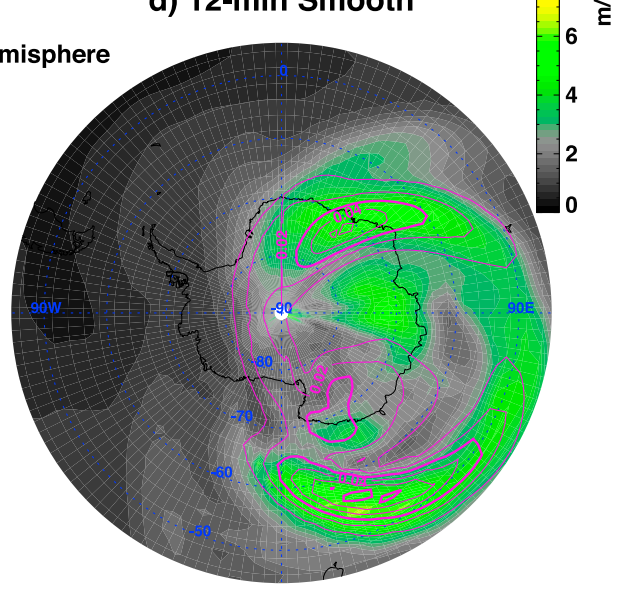

Figure 7. Polar stereographic projections of vertical wind temporal variability (i.e., standard deviations, $\left.\sigma_{w}\right)$ at $285 \mathrm{~km}$ in $\mathrm{m} \mathrm{s}^{-1}$ within the two-hour period (1600-1800 UT) on 7 April 2008 shown in the previous figures for the $(a, b)$ Northern and $(c, d)$ Southern Hemispheres, for the no smoothing (Figures 7a and $7 \mathrm{c}$ ) and 12-minute smoothing (Figures $7 \mathrm{~b}$ and $7 \mathrm{~d}$ ) of the IMF, solar wind and density data. All projections are plotted with respect to the same color scale. Two-hour mean Joule heating in $\mathrm{K} \mathrm{s}^{-1}$ is overplotted with purple lines with $0.01 \mathrm{~K} \mathrm{~s}^{-1}$ contour intervals. Levels $0.03,0.06,0.09$, and $0.12 \mathrm{~K} \mathrm{~s}^{-1}$ are shown with thicker lines.

oval, suggesting that heating by auroral particle precipitation, besides Joule heating, plays an essential role in shaping the vertical wind variability.

[26] Figure 8 shows the polar stereographic projections of the variability of the zonal ion speed simulated by the no smoothing and 12-minute smoothing cases in the Northern and Southern Hemispheres demonstrated in a similar way as in Figure 7, but electron number density in $\mathrm{m}^{-3}$ is overplotted with logarithmically scaled contour lines instead of the Joule heating. Smoothing the IMF greatly reduces the variability of the zonal ion flows. For example, in the Northern Hemisphere, peak value of $\sim 350 \mathrm{~m} \mathrm{~s}^{-1}$ at around $0^{\circ}$ longitude drops to $\sim 250 \mathrm{~m} \mathrm{~s}^{-1}$. The spatial distribution and the magnitude of the zonal ion flow variabilities are asymmetric between the two hemispheres.

[27] Meridional ion flow variability demonstrated in Figure 9 behaves similarly to the zonal ion flow variability: Smoothing weakens the ion flow variability and there is a distinct asymmetry between the two hemispheres.
[28] Studying Figures 7 to 9 suggests that vertical wind variability is enhanced in regions of large Joule heating, where ion flow variability is relatively large and where there is a significant amount of ionization.

\subsection{Wave Structures in the Auroral Thermosphere-Ionosphere}

[29] Next, possible wave-like structures are examined in the investigated auroral latitude sector $\left(68.75^{\circ} \mathrm{S}\right.$ and $78.75^{\circ} \mathrm{E}$ ) at $285 \mathrm{~km}$ for the different degrees of variability in the IMF driver and solar wind data. First, for purposes of completeness of analysis, the temporal variations of the neutral vertical winds are presented again from 1600 to 1800 UT on 7 April in Figure 10a, as had been shown in Figure 4 . The black and red lines denote the no smoothing and the 12-minute smoothing simulations, respectively. The model simulation with the high-resolution IMF and solar wind data show remarkable temporal variability, while the simulation with 12-minute smoothed IMF and solar wind 


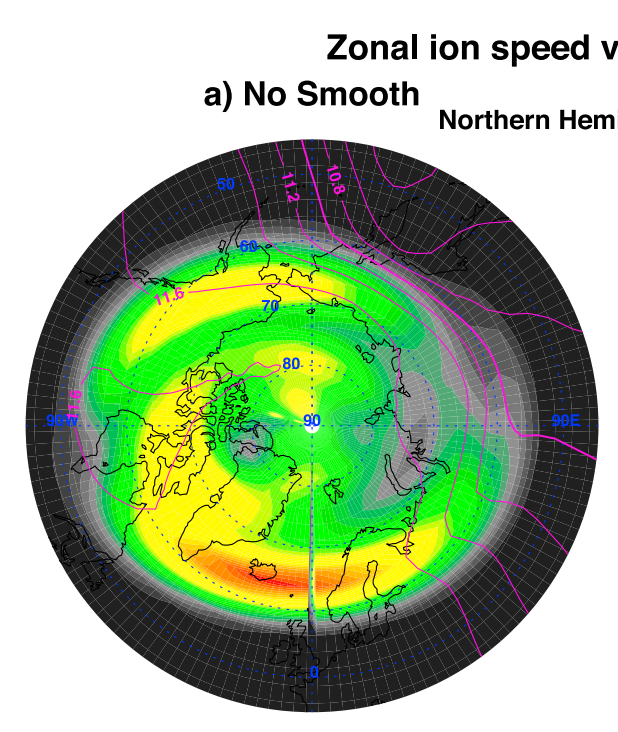

c) No Smooth

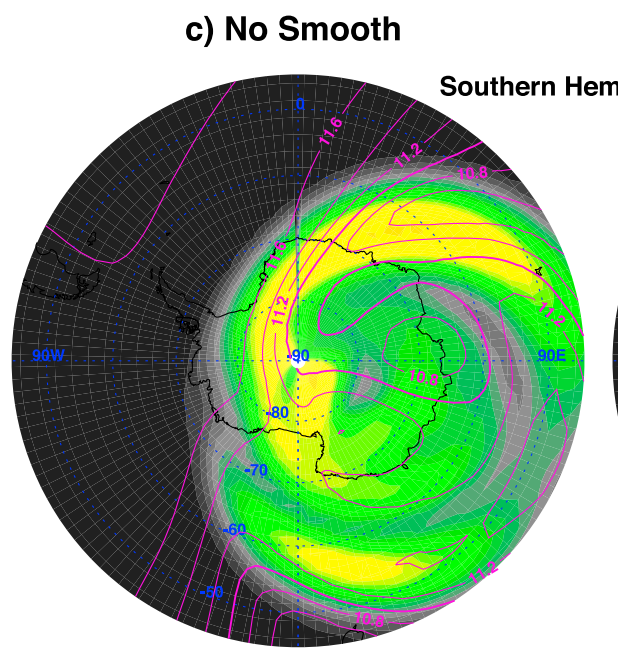

variability, $285 \mathrm{~km}$

b) 12-min Smooth

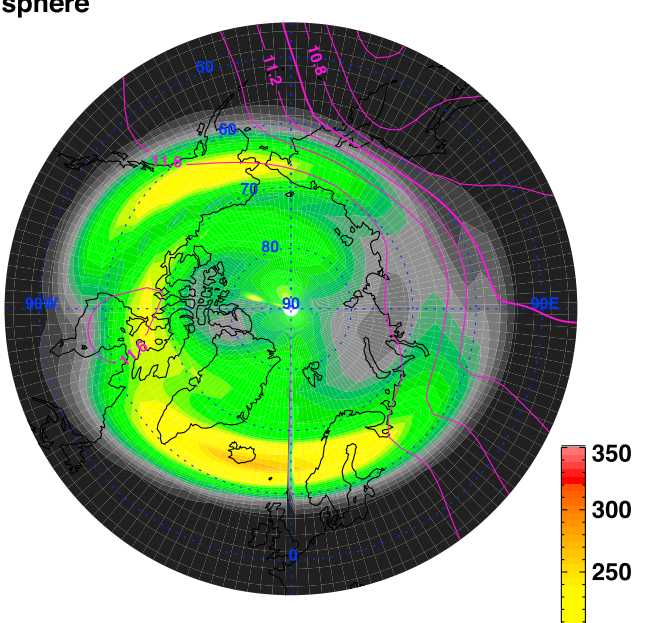

d) 12-min Smooth

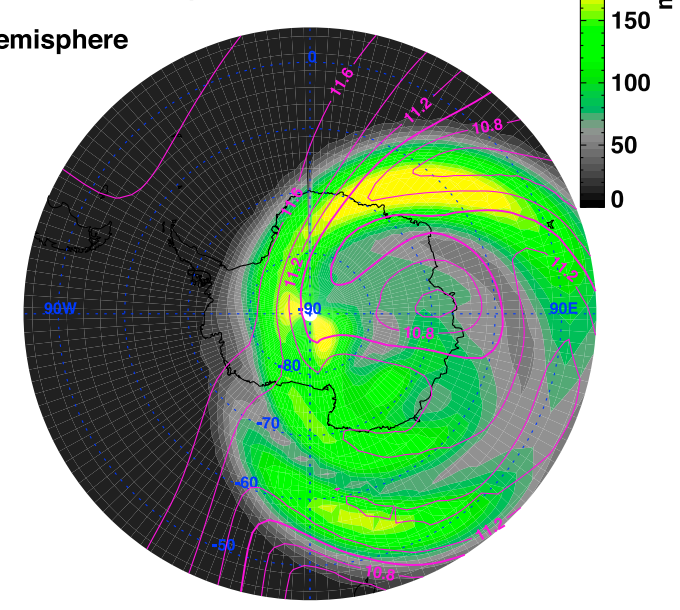

Figure 8. Same as Figure 7 but for the zonal ion speeds. Electron density distribution in $\mathrm{m}^{-3}$ is shown in purple contour lines in logarithmic scale with intervals of 0.2 , where the contour lines for the values 10 , 11 , and 12 are thicker.

data produce much smoother vertical wind variations within the shown time period.

[30] Fourier transform gives information on the frequency content of a temporally variable parameter. Figure $10 \mathrm{~b}$ presents the fast Fourier transform (FFT) of the vertical winds shown in Figure 10a. The Fourier wave amplitudes in $\mathrm{m} \mathrm{s}^{-1}$ are shown as a function of the frequency $\left(\mathrm{s}^{-1}\right)$, where the range of the $\mathrm{x}$-axis is scaled to focus on the high-frequency results from $0.001 \mathrm{~s}^{-1}(\approx 16.6 \mathrm{~min})$ to $0.006 \mathrm{~s}^{-1}(\approx 2.7 \mathrm{~min})$. High time resolution of the model and the data output enable the investigation of wave period as short as a few minutes. Two distinct maxima are seen in the wave amplitude: $\sim 0.7 \mathrm{~m} \mathrm{~s}^{-1}$ at $10 \mathrm{~min}$ period and $\sim 0.9 \mathrm{~m} \mathrm{~s}^{-1}$ at $5.2 \mathrm{~min}$ period that are highlighted in green.

[31] Although Fourier transform provides overall information on the importance of a fluctuation in a given period of time, it does not provide detailed information about how various wave modes evolve in time. Wavelet analysis can provide insight into the different modes of variability and how they evolve in time, that is, how the frequency content changes in time. In order to determine the temporal evolution of high-frequency wave structures, wavelet analysis of the vertical wind data shown in Figure 10a is performed. Wavelet analysis is a useful tool to investigate wave generation processes and time evolution of wave activity and has been applied to study gravity wave signatures in the atmosphere [Zink and Vincent, 2001]. Figures $10 \mathrm{c}$ and $10 \mathrm{~d}$ present the period-time distribution of the normalized wavelet power spectral energy density (PSD) within the 2-hour period for the no smoothing and the 12-minute smoothing simulations, respectively. The same contour color scaling has been used to enable a comparison between the two simulations. Remarkable wave structures are revealed in the no smoothing case that are much weaker in the 12-minute smoothing simulation. When high-resolution IMF and solar wind data are used in GITM, waves with periods $\mathcal{T}$ of 5-6 min and 10-11 min are prevalent, occurring several times in the shown time period. In particular, the wave activity with $\mathcal{T} \approx 5$ min centered around 1710 UT has the peak strength. The wave events seen in the no smoothing case are dramatically weakened when the model is run with 12-minute smoothed IMF and solar wind data and in fact 


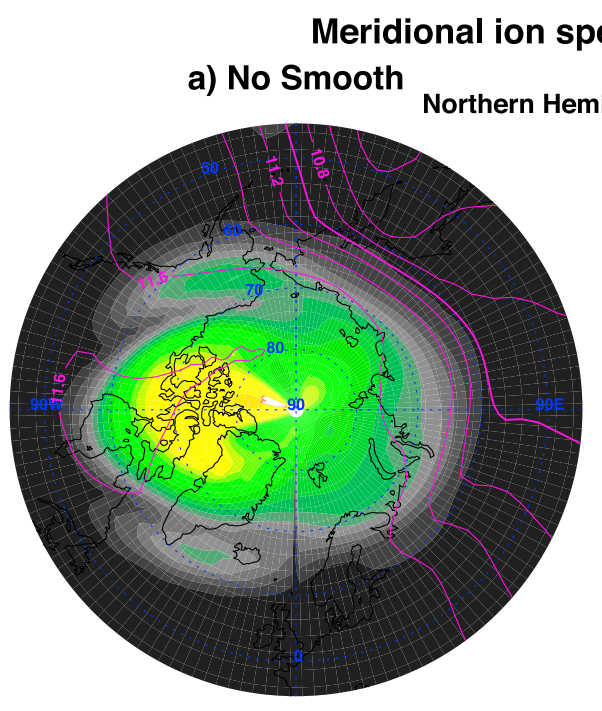

c) No Smooth

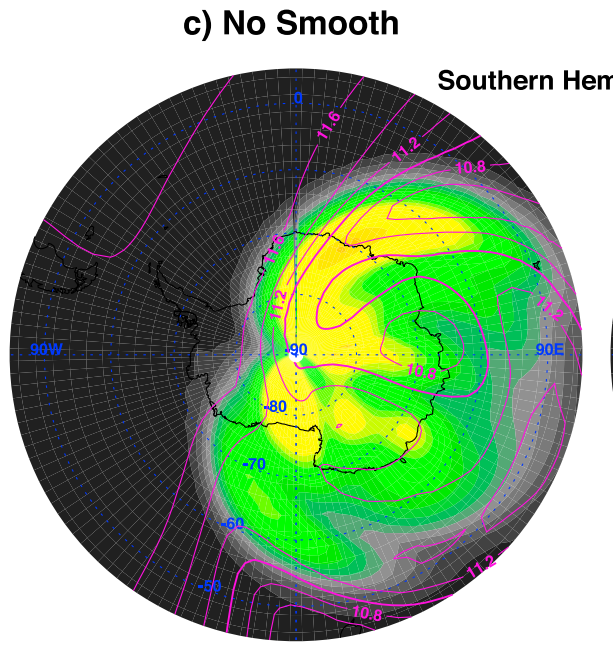

eed variability, $285 \mathrm{~km}$

b) 12-min Smooth

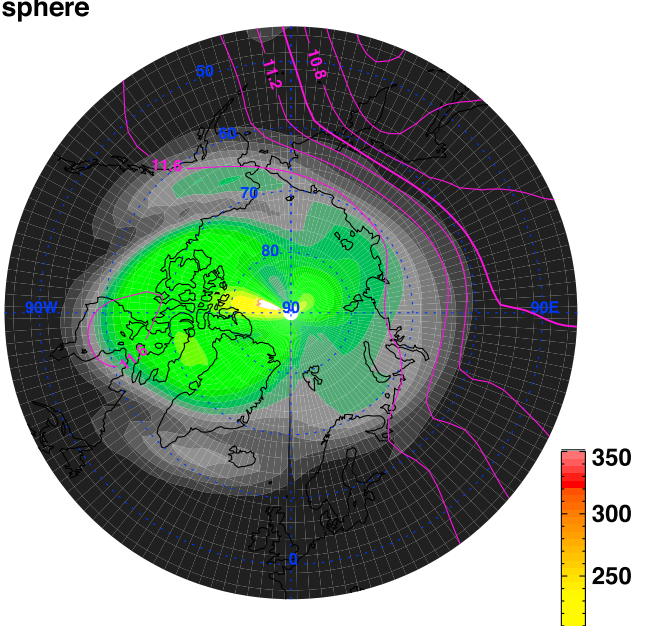

d) 12-min Smooth

Figure 9. Same as Figure 8 but for the meridional ion speeds.

partially disappear. These results support the general FFT trend shown in Figure 10b, where FFT values provide a mean value for the 2-hour period for the wave activity, while the wavelet analysis can demonstrate a more detailed information in the form of time-frequency (period) distribution.

[32] Because a substantial dynamical and thermal link has been seen between the Joule heating and vertical winds in the results presented in the previous sections, the same wave analysis is performed for the Joule heating as well. This is shown in Figure 11 in the same manner as in Figure 10. The no smoothing run produces much larger $Q_{J}$ than the run with smoothed IMF, and it demonstrates rapid temporal variations as seen before. Figure $11 \mathrm{~b}$ presents the FFT of the Joule heating data seen in Figure 11a. The high-resolution IMF and solar wind data simulation is characterized by much larger amplitude wave structures in Joule heating. Figures $11 \mathrm{c}$ and $11 \mathrm{~d}$ compare the wavelet analysis of the Joule heating data for the no smoothing and 12-minute smoothing simulations. The high-resolution data run demonstrates enhanced wave activity of 5-, 10-, and 15-minute wave periods that are dramatically weakened in the run with smoothed IMF and solar wind input data. Finally, intercomparison of the wavelet analysis of vertical wind and Joule heating data reveal some degree of correlation between the time period distribution of wave activity seen in $w_{n}$ and $Q_{J}$. Especially, in the enhanced wave event with $\mathcal{T} \approx 5$ min seen in the vertical wind data centered at 1710 UT (70 min mark in the figure), is seen in the Joule heating at the same time.

[33] The wave events of $\mathcal{T} \approx 5 \mathrm{~min}$ and $\mathcal{T} \approx 10 \mathrm{~min}$ are characteristic of acoustic-gravity waves. As gravity waves and acoustic (sound) waves represent the low-frequency and high-frequency branch of the acoustic-gravity wave spectrum, they can be identified in the model according to their periods extracted from the wavelet analysis. The acoustic wave speed $c_{s}=\gamma R T$ is about $810 \mathrm{~m} \mathrm{~s}^{-1}$ at around $285 \mathrm{~km}$, where $\gamma$ is the adiabatic constant, $R$ is the gas constant and $T$ is the neutral temperature. The Brunt-Väisälä (buoyancy) frequency $N=\left[(\gamma-1)\left(g^{2} / c_{s}^{2}\right)\right]^{1 / 2}$ is then $\sim 0.009 \mathrm{rad} \mathrm{s}^{-1}$. For the $\mathcal{T} \approx 5$ min wave, we have $\omega=0.02 \mathrm{~s}^{-1}>2 N$, which is typical of an acoustic wave. For the $\mathcal{T} \approx 10$ min wave, we have $\omega=0.01 \mathrm{~s}^{-1} \approx N$, which is more typical of a gravity wave.

[34] The buoyancy frequency, $N$, is the eigenfrequency (resonant frequency), $\omega_{e}$, of an oscillation of a vertically 

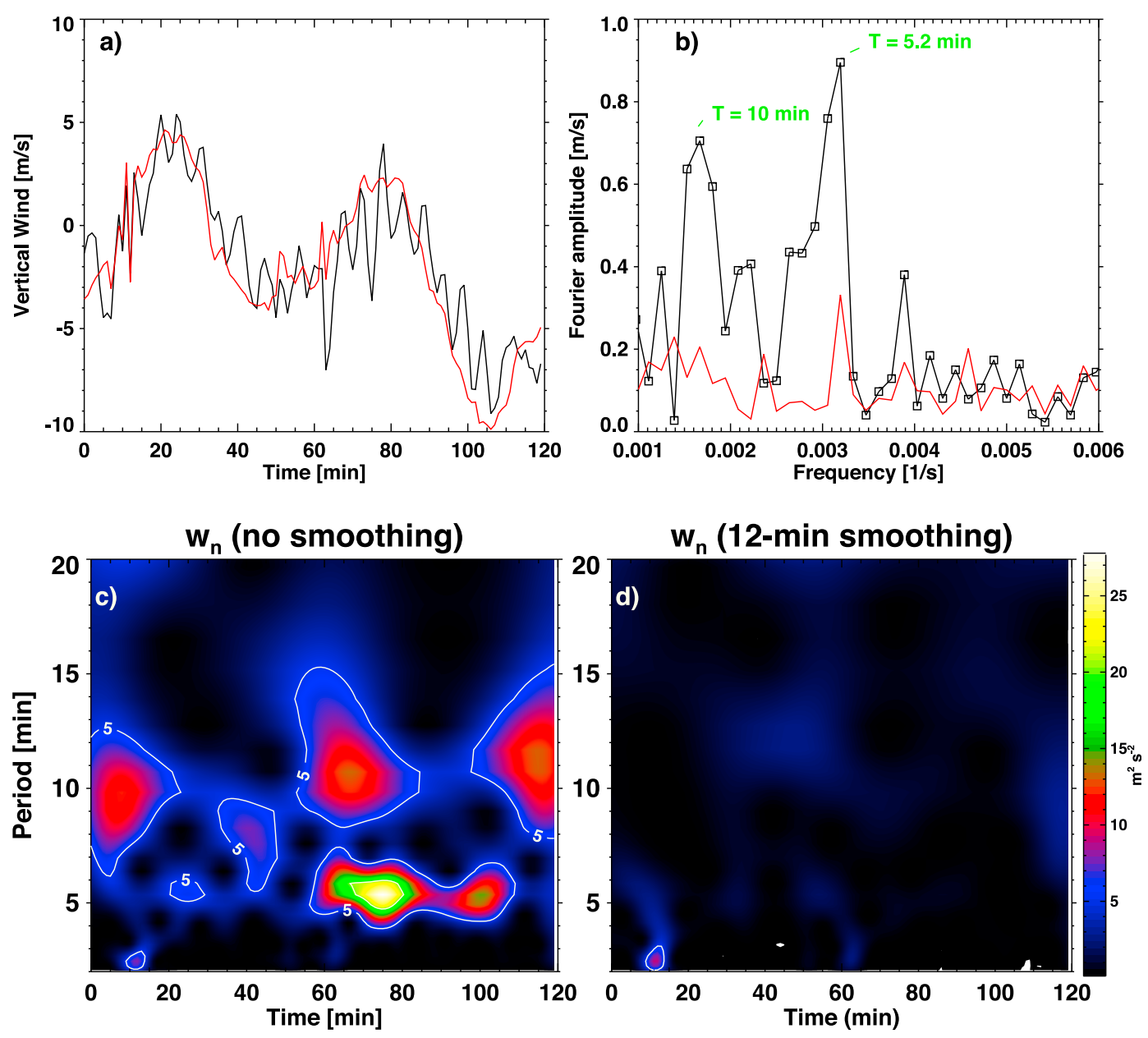

Figure 10. Analysis of high-frequency wave structures in the vertical winds at $285 \mathrm{~km}$ for $68.75^{\circ} \mathrm{S}$, $78.75^{\circ} \mathrm{E}$ from 1600 to 1800 UT on 7 April: (a) temporal variation of the vertical wind in the 2-hour period for the no smoothing (black) and 12-minute smoothing (red) cases and (b) fast Fourier transform (FFT) of the vertical wind data shown in Figure 10a. The peak values are highlighted in green for the 10 min and 5.2 min waves. Wavelet analysis of the vertical wind data in the form of normalized power spectral density is shown for the (c) no smoothing and (d) 12-minute smoothing cases.

displaced air parcel in a stably stratified adiabatic fluid. If the (angular) frequency $\omega$ of an external forcing is close to the resonant frequency, i.e., $\omega \approx \omega_{e}$, then the amplitude of oscillation is largest. In general, various dynamical and thermal processes generate waves in the atmosphere. In principle, these are forced oscillations. The amplitude, thus the energy, of an oscillation strongly depends on the difference between the applied frequency and the eigenfrequency of a system. Note that $N$ is not constant in the atmosphere and varies significantly, in particular, with altitude [e.g., Yiğit and Medvedev 2010, Figure 1d]. So, for $\omega \approx N$ a strong spectral peak is expected, however, the intensity of this peak also depends on wave damping (dissipation). At those altitudes in the ionosphere-thermosphere, ion drag, thermal conduction and molecular viscosity are the dominant dissipation processes for internal gravity waves [Klostermeyer, 1972; Yiğit et al., 2009] and can substantially limit wave amplitudes.

[35] These results suggest that the magnitude of heliospheric variability has a great impact on the occurrence of wave structures in thermospheric vertical winds, which can impact vertical wind variability.

\section{Discussion and Conclusions}

[36] In this study, using the University of Michigan 3-D time-dependent Global Ionosphere Thermosphere Model (GITM), the variability, magnitude, and generation mechanisms of thermospheric neutral vertical winds have been investigated, implementing observed real-time variations of the Interplanetary Magnetic Field (IMF), solar wind parameters, solar and auroral activity as model input. GITM was systematically run 3 times for the period 6 to 8 April 2008, first without smoothing the high-resolution real-time IMF and solar wind input data, then in the subsequent two runs 5-minute and 12-minute smoothed IMF and solar wind data are used. The temporal variations of thermospheric parameters based on high-time resolution of 1-minute outputs for the period of 1600 to 1800 UT on 7 April 2008 have been studied. This period of time has also been studied observationally by Anderson et al. [2011]. Their study had 

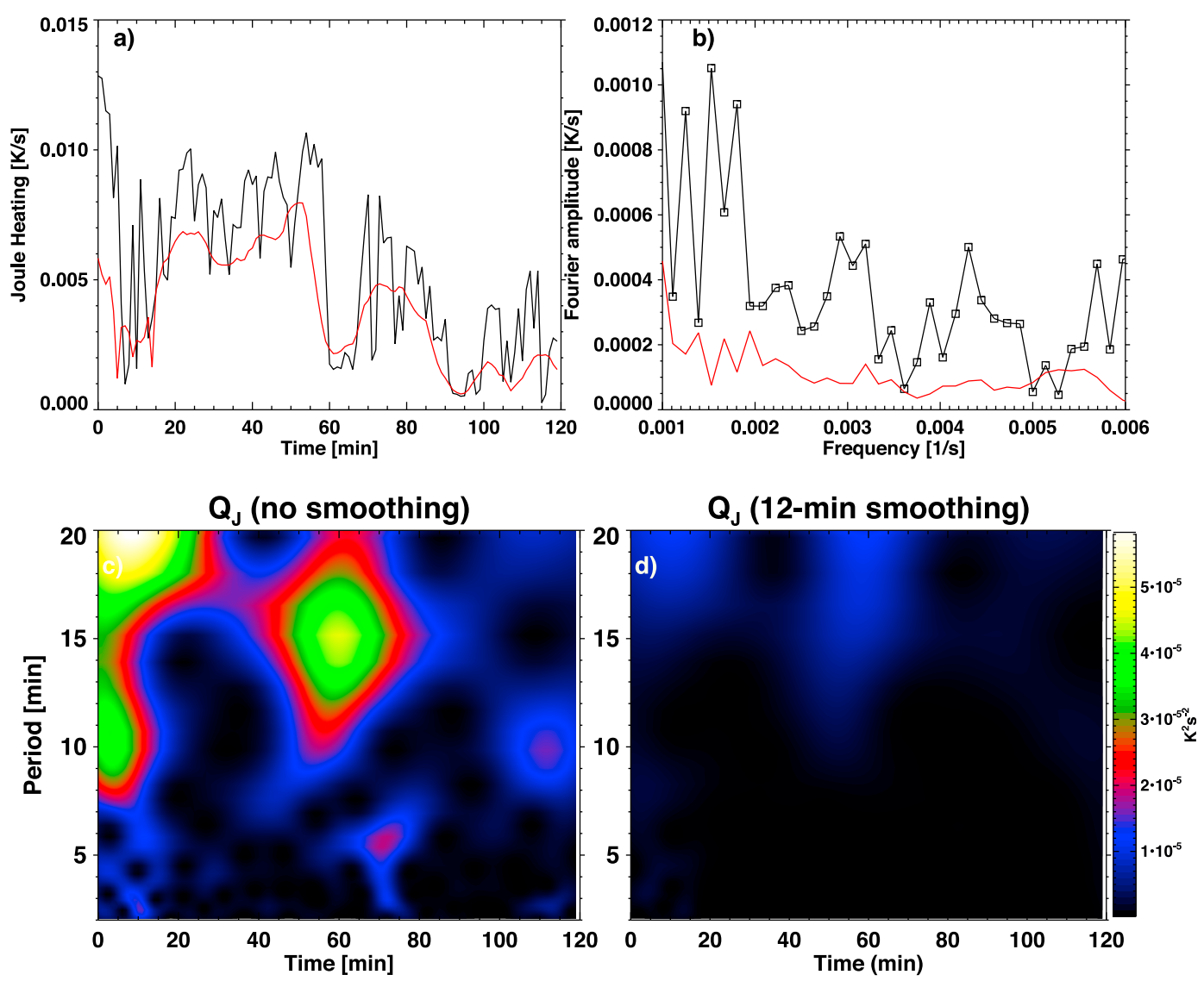

Figure 11. Same as Figure 10 but for the Joule heating.

suggested that horizontal wind divergence greatly shapes the vertical winds in the thermosphere, which the presented work has investigated in further detail by looking at what the role of the interplay between different thermospheric parameters is in influencing the magnitude and the variability of vertical winds.

[37] A number of authors have studied the relationship between the vertical winds and the divergence of the horizontal winds [Burnside et al., 1981; Biondi, 1984; Crickmore, 1993; Cooper and Conde, 2006; Cooper et al., 2009]. The advantages of a nonhydrostatic model such as GITM in studying thermospheric vertical winds and their variability are, among others, (1) high model time resolution of 2-4 s, (2) the individual vertical momentum flux deposition terms can be investigated in detail, (3) the significance of nonhydrostatic acceleration can be quantified, (4) the temporal variations of the horizontal wind divergence can be compared with that of the vertical winds and other thermospheric parameters. In order to investigate the relationship between the vertical winds and horizontal wind divergence and high-latitude heat sources and ion flows, the current study benefited from all four aspects.

[38] The magnitude and the orientation of the IMF greatly shapes the ionospheric convection electric fields and the associated ion flows [Weimer, 1996]. By assuming idealistic IMF input variations along with constant moderate solar activity and auroral activity, Yiğit and Ridley [2011a] demonstrated that nonhydrostatic acceleration is an important source of momentum flux deposition in the thermosphere, especially when ion flow magnitude and variability are enhanced. Primarily, the findings of Yigit and Ridley [2011a] along with the recent observations of Anderson et al. [2011] have motivated the study presented in this paper.

[39] If our modeling results are investigated in relatively small timescales of minutes, horizontal divergence is not able to drive the observed small-scale variations of the vertical winds. Horizontal winds vary much more slowly than the other thermospheric parameters, such as the Joule heating and auroral heating, ion flows, and vertical winds. However, over larger timescales, horizontal wind divergence can substantially impact the magnitude of vertical winds via continuity. The Burnside relation [Burnside et al., 1981] that relates horizontal wind divergence to vertical wind is valid to a much lesser extent in the high-latitudes. Similarly, other studies have found that the Burnside relation can, in fact, break down under strong disturbances in the high-latitudes [Crickmore, 1993; Smith and Hernandez, 1995]. For instance, using a time-dependent three-dimensional local model of the thermosphere, Cooper et al. [2009] have investigated the applicability of the Burnside et al. [1981] relation. They have found that although the Burnside et al. [1981] condition is usable under slowly varying conditions above the $F$ region peak, it is applicable to a limited extent when the high-latitude forcing is varying rapidly in time. Similar to what their results imply, our modeling study indicates that horizontal divergence is not always capable of driving small-scale vertical wind variability under highly variable high-latitude conditions at $F$ region altitudes. 
[40] Variations of the IMF and how they are implemented in the model greatly impact the magnitude and the variability of the neutral vertical winds even under quiet geomagnetic and solar conditions, as is the case in the chosen period of time. In this study, by systematically performing temporal smoothing of the observed real-time IMF and solar wind parameters, the importance of the IMF variations for the variability and magnitude of vertical winds has been studied. If the temporal variability of the IMF is reduced by data smoothing, then ion flows are less variable while neutral winds are affected to a much lesser extent due to their larger inertia. Also, the neutral wind divergence varies much slower in time compared with other thermospheric parameters that effect the neutral winds. Nonhydrostatic acceleration is a significant source of momentum flux deposition and is overall dependent in a complex manner on the magnitude and the temporal variability of the IMF, ion flows, and Joule and auroral heating. It can substantially shape the small-scale temporal variability of thermospheric vertical winds. The lack of proper representation of small-scale temporal structures of the IMF and solar wind parameters is one possible mechanism that can be responsible for the underestimation of the magnitude and temporal variability of thermospheric vertical winds in general circulation models.

[41] As discussed in Bristow [2008, and references therein], the magnetosphere is a region of complex dynamics that couples to the high-latitude ionosphere driving plasma flows (convection) that can exhibit highly variable fluctuations. Variable plasma structures of different scale sizes are systematically detected by observations [Ridley and Clauer, 1996; Kivanç and Heelis, 1998]. Besides investigating the general question of the origin of variability in the ionosphere-thermosphere, Bristow [2008] examined the level of variability in plasma flows in the high-latitude ionosphere based on 6 years of Super Dual Auroral Radar Network (SuperDARN) data. His analysis showed that zonal and meridional ion flows can possess a typical variability (standard deviation) of up to $350-400 \mathrm{~m} \mathrm{~s}^{-1}$ in the high-latitudes. Our results presented in Figures 8 and 9 for zonal and meridional ion flow variabilities respectively are in good qualitative agreement with his observational values. For example, large standard deviations of zonal and meridional ion flows are seen around polar latitudes in observations, which the model suggests as well. It is important to note that Bristow [2008] analysis is based on a large number of data sets of 6 years, while the model analysis focuses on the nighttime only. The model underestimates the peak values of ion flows to some extent, which could be due to the lack of a self-consistent magnetosphere in our modeling framework, which means that the internal magnetospheric processes are not accounted for properly. Also, the modeling work focuses solely on the variability in the IMF driver and the solar wind parameters. A possible source of variability that is not captured by this study or by Bristow [2008] is the internal waves of lower atmospheric origin that can directly propagate into the thermosphere-ionosphere system from the tropospherestratosphere [Yiğit et al., 2009, 2012]. A further possible interpretation of our results is that lack of representation of internal magnetospheric processes can lead to underestimation of vertical wind variability as they affect plasma flow variability, which can substantially drive vertical winds.
[42] Comprehensive modeling studies can offer an alternative perspective of vertical wind variability compared to detailed long-term observations. For example, the spatial structures of vertical wind variability shown Figure 7 in terms of standard deviations of vertical wind within twohour period is dramatically different to the large-scale average maps of vertical wind variability derived from Dynamic Explorer-2 Wind and Temperature Spectrometer vertical wind data presented in the works by Innis and Conde [2001, 2002]. This is because these authors have used a very large data set to evaluate average spatial distribution of vertical wind variability, which they referred to as "vertical wind activity maps", while the present study focused on a twohour universal time period at a lower altitude that is close to Fabry-Perot measurements conducted by Anderson et al. [2011].

[43] Innis and Conde [2001] and Innis and Conde [2002] have found no significant hemispheric differences in average vertical wind variability while the present study demonstrates distinct hemispheric differences in the spatial structure and magnitude of vertical wind temporal variability. This suggests that on relatively short timescales, spatial structure of vertical wind temporal variability can be different to variations on larger timescales.

[44] The variability of spatial structures that can be simulated by the model is limited by the use of empirical electric potential field and particle precipitation models. So, while using high-time resolution real-time IMF data leads to increased temporal variability of ion convection, the spatial variations of ion flow patterns are still underestimated because empirical models can represent the spatial variability to some degree. Hence, driving GCMs with empirical models that use high-time resolution IMF data improves the representation of upper atmospheric variability to a limited extent.

[45] This work has also investigated wave structures in the high-latitude ionosphere. Wavelet analysis has been applied on the vertical wind data between 1600 and 1800 UT on 7 April in order to determine relatively high-frequency wave structures. This analysis is done both for simulations with no smoothing in the IMF and solar wind data and for the 12-minute smoothing case to demonstrate how the IMF variations and their implementation in GCMs can impact simulations of wave structures. In the no smoothing case, high-frequency wave structures with 5-6 minute and 10-11 minute periods are prevalent in the studied period. These periods are characteristic of acoustic-gravity waves. When the model is run with 12-minute smoothed IMF and solar wind data, these wave structures weaken dramatically. A number of authors considered the relation between atmospheric gravity waves and vertical winds in the midlatitudes [Hernandez, 1982] and high-latitudes [Rees et al., 1984b; Johnson et al., 1995; Innis and Conde, 2001, 2002]. Deng et al. [2008] simulated for the first time an acoustic wave in a global model and demonstrated in an idealized run that the associated perturbations can impact vertical winds dramatically. They found similar wave periods as the ones demonstrated in this work. Also, Anderson et al. [2011] suggest that the time evolution of thermospheric vertical winds should be observed at multiple locations in future studies to investigate gravity wave signatures in more detail. The current study suggests that the variability of the 
IMF driver and the solar wind data have great impact on the simulation of acoustic-gravity wave structures in the auroral thermosphere. Thus, underestimation of acousticgravity wave activity in global models is a possible contributor to why vertical wind magnitude and variability are underestimated.

[46] Note that an explicit quantification of variability is strongly dependent on what temporal and spatial scales are analyzed. A broad spectrum of fluctuations (wave structures) are present in the upper atmosphere due to coupling to the magnetosphere and to lower atmospheric sources. This makes the thermosphere-ionosphere an interesting region to study various scales of variability. There is an increasing number of studies investigating turbulence in the auroral ionosphere [e.g., Kozelov et al., 2008; Lund, 2010].

[47] The modeling study presented here has provided further insight into the connection between the IMF, ion flows, Joule heating, and vertical wind variability. However, the magnitude of the vertical winds observed by Anderson et al. [2011] are still underestimated by GITM. The model uses empirical models for implementing auroral activity and electric fields. This is a possible reason for this underestimation. Future modeling studies will consider the importance of electric field variability on the magnitude and variability of Joule heating and neutral vertical winds. Electric field variability is expected to impact the Joule heating dramatically [Matsuo and Richmond, 2008] and can thus influence the magnitude and variability of thermospheric vertical winds. Also, the effect of model spatial resolution on the morphology of wave structures and on the temporal variation of thermospheric parameters will be investigated.

[48] Acknowledgments. Erdal Yiğit and Aaron J. Ridley were supported by the following grants: NSF grants (ANT0838828, ATM0640429, ATM069336, and AGS-1103333) and AFOSR grant FA9550-07-1-0434. Mark B. Moldwin was partially supported by NASA grant NNX10AQ53G. The Global Ionosphere Thermosphere Model simulations have been performed using the computer facilities at the Department of Atmospheric and Oceanic and Space Sciences of the University of Michigan.

[49] Robert Lysak thanks Peter Dyson and another reviewer for their assistance in evaluating this paper.

\section{References}

Anderson, C., T. Davies, M. Conde, P. Dyson, and M. J. Kosch (2011), Spatial sampling of the thermospheric vertical wind field at aurora latitudes, J. Geophys. Res., 116, A06320, doi:10.1029/2011JA016485.

Biondi, M. A. (1984), Measured vertical motion and converging and diverging horizontal flow of the midlatitude thermosphere, Geophys. Res. Lett., 11(1), 84-87.

Bristow, W. (2008), Statistics of velocity fluctuations observed by SuperDARN under steady interplanetary magnetic field conditions, J. Geophys. Res., 113, A11202, doi:10.1029/2008JA013203.

Burnside, R. G., F. A. Herrero, J. W. Meriweather Jr., and J. C. G. Walker (1981), Optical observations of thermospheric dynamics at Arecibo, J. Geophys. Res., 86, 5532-5540.

Conde, M., and P. L. Dyson (1995), Thermospheric vertical winds above Mawson, Antarctica, J. Atmos. Terr. Phys., 57, 589-596.

Cooper, S. L., and M. Conde (2006), Origins of horizontal divergence in the auroral thermosphere: A modeling study, Geophys. Res. Lett., 33, L21111, doi:10.1029/2006GL027601.

Cooper, S. L., M. Conde, and P. Dyson (2009), Numerical simulations of thermospheric dynamics: Divergence as a proxy for vertical winds, Ann. Geophys., 27, 2491-2502.

Crickmore, R. I. (1993), A comparison between vertical winds and divergence in the high-latitude thermoshere, Ann. Geophys., 11, 728-733.

Deng, Y., A. D. Richmond, A. J. Ridley, and H. Liu (2008), Assessment of the non-hydrostatic effect on the upper atmosphere using a general circulation model (GCM), Geophys. Res. Lett., 35, L01104, doi:10.1029/2007GL032182.

Dickinson, R. E., E. C. Ridley, and R. G. Roble (1981), A threedimensional general circulation model of the thermosphere, J. Geophys. Res., 86(A3), 1499-1512.

Fuller-Rowell, T. J., and D. S. Evans (1987), Height-integrated Pederson and Hall conductivity patterns inferred from TIROS-NOAA satellite data, J. Geophys. Res., 92, 7606-7618.

Fuller-Rowell, T. J., and D. Rees (1980), A three dimensional timedependent global model of the thermosphere, J. Atmos. Sci., 37, 2545-2567.

Hedin, A. E. (1991), Extension of the MSIS thermosphere model into the middle and lower atmosphere, J. Geophys. Res., 96, 1159-1172.

Heppner, J. P., M. C. Liebrecht, N. C. Maynard, and R. F. Pfaff (1993), High-latitude distributions of plasma waves and spatial irregularities from DE2 alternating current elec-tric field observations, J. Geophys. Res., 98 1629-1652.

Hernandez, G. (1982), Vertical motions of the neutral thermosphere at midlatitude, Geophys. Res. Lett., 9(5), 555-557.

Innis, J. L., and M. Conde (2001), Thermospheric vertical wind activity maps derived from Dynamics Explorer-2 WATS observations, Geophys. Res. Lett., 28, 3847-3850.

Innis, J. L., and M. Conde (2002), High-latitude thermospheric vertical wind activity from Dynamics Explorer 2 Wind and Temperature Spectrometer observations: Indications of a source region for polar cap gravity waves, J. Geophys. Res., 107(A8), 1172, doi:10.1029/2001JA009130.

Innis, J. L., P. A. Greet, and P. L. Dyson (1996), Fabry-Perot spectrometer observations of the auroral oval/polar cap boundary above Mawson, Antarctica, J. Atmos. Terr. Phys., 58, 1973-1988.

Innis, J. L., P. Greet, D. Murphy, M. G. Conde, and P. Dyson (1999), A large vertical wind in the thermosphere at the auroral oval/polar cap boundary seen simultaneously from mawson and davis, antarctica, J. Atmos. Sol. Terr. Phys., 61, 1047-1058.

Ishii, M., M. Conde, R. W. Smith, M. Krynicki, E. Sagawa, and S. Watari (2001), Vertical wind observations with two fabry-perot interferometers at poker flat, alaska, J. Geophys. Res., 106, 10,537-10,551.

Johnson, E. S., and R. A. Heelis (2005), Characteristics of ion velocity structure at high latitudes during steady southward interplanetary magnetic field conditions, J. Geophys. Res., 110, A12301, doi:10.1029/ 2005JA011130.

Johnson, F. S., W. B. Hanson, R. R. Hodges, W. R. Coley, G. R. Carignan, and N. W. Spencer (1995), Gravity waves near $300 \mathrm{~km}$ over the polar cap, J. Geophys. Res., 100, 23,993-24,002.

Kil, H., Y.-S. Kwak, L. J. Paxton, R. R. Meier, and Y. Zhang (2011), O and $\mathrm{N}_{2}$ disturbances in the $\mathrm{F}$ region during the 20 November 2003 storm seen from TIMED/GUVI, J. Geophys. Res., 116, A02314, doi:10.1029/ 2010JA016227.

Kivanç, Ö., and R. A. Heelis (1998), Spatial distribution of ionospheric plasma and field structures in the high-latitude $F$ region, $J$. Geophys. Res., 103, 6955-6968.

Klostermeyer, J. (1972), Influence of viscosity, thermal conduction, and ion drag on the propagation of atmospheric gravity waves in the thermosphere, Z. Geophys., 38, 881-890.

Kozelov, B. V., I. V. Golovchanskaya, A. A. Ostapenko, and Y. V. Fedorenko (2008), Wavelet analysis of high-latitude electric and magnetic fluctuations observed by the Dynamic Explorer 2 satellite, J. Geophys. Res., 113, A03308, doi:10.1029/2007JA012575.

Lund, E. J. (2010), On the dissipation scale of broadband ELF waves in the auroral region, J. Geophys. Res., 115, A01201, doi:10.1029/ 2009JA014545.

Matsuo, T., and A. D. Richmond (2008), Effects of high-latitude ionospheric electric field variability on global thermospheric Joule heating and mechanical energy transfer rate, J. Geophys. Res., 113, A07309, doi:10.1029/2007JA012993.

Matsuo, T., A. D. Richmond, and K. Hensel (2003), High-latitude ionospheric electric field variability and electric potential derived from DE-2 plasma drift measurements: Dependence on IMF and dipole tilt, J. Geophys. Res., 108(A1), 1005, doi:10.1029/2002JA009429.

Price, G. D., R. W. Smith, and G. Hernandez (1995), Simultaneous measurements of large vertical winds in the upper and lower thermosphere, J. Atmos. Terr. Phys., 57, 631-643.

Rees, D., R. W. Smith, P. J. Charleton, F. G. McCormac, N. Lloyd, and A. Kesteen (1984a), The generation of vertical thermospheric winds and gravity waves at auroral latitudes-I. observations of vertical winds, Planet. Space Sci., 32, 667-684.

Rees, D., R. W. Smith, and R. Gordon (1984b), The generation of vertical thermospheric winds and gravity waves at auroral latitudes-II. theory and numerical modelling of vertical winds, Planet. Space Sci., 32, 685-705. 
Richmond, A. D., E. C. Ridley, and R. G. Roble (1992), A thermosphere/ ionosphere general circulation model with coupled electrodynamics, Geophys. Res. Lett., 19, 601-604.

Ridley, A. J., and C. R. Clauer (1996), Characterization of the dynamic variations of the dayside high-latitude ionospheric convection reversal boundary and relationship to interplanetary magnetic field orientation, J. Geophys. Res., 101(A5), 10,919-10,938.

Ridley, A. J., D. L. D. Zeeuw, T. I. Gombosi, and K. Powell (2001), Using steady-state mhd results to predict the global state of the magnetosphereionosphere system, J. Geophys. Res., 106, 30,067-30,076.

Ridley, A. J., Y. Deng, and G. Tóth (2006), The global ionospherethermosphere model, J. Atmos. Sol. Terr. Phys., 68, 839-864.

Roble, R. G., E. C. Ridley, and A. D. Richmond (1988), A coupled thermosphere/ionosphere general circulation model, Geophys. Res. Lett., 15(12), $1325-1328$.

Rodger, A. S., G. D. Wells, R. J. Moffett, and G. J. Bailey (2001), The variability of joule heating, and its effects on the ionosphere and thermosphere, Ann. Geophys., 19, 773-781.

Smith, R. W., and G. Hernandez (1995), Vertical winds in the thermosphere within the polar cap, J. Atmos. Terr. Phys., 57, 611-620.

Spencer, N. W., R. F. Theis, L. E. Wharton, and G. R. Carignan (1976), Local vertical motions and kinetic temperature from AE-C as evidence for aurora-induced gravity waves, Geophys. Res. Lett., 3, 313-316.

Wang, W., T. L. Killeen, A. G. Burns, and R. G. Roble (1999), A highresolution, three-dimensional, time-dependent, nested grid model of the coupled thermosphere-ionosphere, J. Atmos. Sol. Terr. Phys., 61, $385-397$.

Wardill, P., and F. Jacka (1986), Vertical motions in the thermosphere over Mawson, Antarctica, J. Atmos. Terr. Phys., 48, 289-292.
Weimer, D. (1996), A flexible, IMF dependent model of high-latitude electric potentials having space weather application, Geophys. Res. Lett., 23, 2549-2552.

Weimer, D. R. D. M. Ober, N. C. Maynard, W. J. Burke, M. R. Collier, D. J. McComas, N. F. Ness, and C. W. Smith (2002), Variable time delays in the propagation of the interplanetary magnetic field, J. Geophys. Res., 107(A8), 1210, doi:10.1029/2001JA009102.

Yiğit, E., and A. S. Medvedev (2010), Internal gravity waves in the thermosphere during low and high solar activity: Simulation study, J. Geophys. Res., 115, A00G02, doi:10.1029/2009JA015106.

Yiğit, E., and A. J. Ridley (2011a), Role of variability in determining the vertical wind speeds and structure, J. Geophys. Res., 116, A12305, doi:10.1029/2011JA016714.

Yiğit, E., and A. J. Ridley (2011b), Effects of high-latitude thermosphere heating at various scale sizes simulated by a nonhydrostatic global thermosphere-ionosphere model, J. Atmos. Sol. Terr. Phys., 73, 592-600, doi:10.1016/j.jastp.2010.12.003.

Yiğit, E., A. S. Medvedev, A. D. Aylward, P. Hartogh, and M. J. Harris (2009), Modeling the effects of gravity wave momentum deposition on the general circulation above the turbopause, J. Geophys. Res., 114, D07101, doi:10.1029/2008JD011132.

Yiğit, E., A. S. Medvedev, A. D. Aylward, A. J. Ridley, M. J. Harris, M. B. Moldwin, and P. Hartogh (2012), Dynamical effects of internal gravity waves in the equinoctial thermosphere, J. Atmos. Sol. Terr. Phys., doi:10.1016/j.jastp.2011.11.014, in press.

Zink, F., and R. A. Vincent (2001), Wavelet analysis of stratospheric gravity wave packets over macquuarie island: 1. Wave parameters, J. Geophys. Res., 106(D10), 10,275-10,288. 